may be encouraged to supervise SHOs taking on appropriate cases within the community mental health teams. At present, the training requirements are not mandatory and are easily overlooked by SHOs, who either lack awareness of the recommendations, have limited access to training or who are dealing with the pressures of the current MRCPsych exams.

With the envisaged modular/workplace-based assessment equivalent to the MRCPsych, perhaps psychotherapy training will become more fully integrated into the system. Surely, experience in psychotherapy, psychodynamic and cognitive-behavioural therapy should be an essential part of training, to help develop listening skills, to better understand our patients and the roots of their problems and to encourage us to manage patients using the biopsychosocial model to the full.

ROYAL COLLEGE OF PSYCHIATRISTS (2003) Executive Summary. Requirements for PsychotherapyTraining as Part of Basic SpecialistTraining. London: Royal College of Psychiatrists. http://www.rcpsych.ac.uk/ traindev/postgrad/ptBasic.pdf

ROYAL COLLEGE OF PSYCHIATRISTS (2005) The Dean's Medical Education Newsletter, April 2005 London: Royal College of Psychiatrists. http:// www.rcpsych.ac.uk/traindev/postgrad/ dean_05.pdf

K. Webb Senior House Officer in Psychiatry, Nottingham Psychotherapy Unit, 114 Thorneywood Mount, Nottingham NG3 2PY

\section{Gaps in adolescent services}

Singh et al (Psychiatric Bulletin, August 2005, 29, 292-294) highlighted problems at the interface between adolescent and adult mental health services. Some years ago in Brisbane (Australia) our adolescent services worked to a strict lower age limit of under 16 and referrals had to be of adolescents living at home and attending school. As an adult community service provider I encountered a young lady in crisis whom I considered required adolescent services - she was 15 and still at school. I phoned the relevant adolescent clinic. The response? So she was 15 and at school, but she had left home so she did not qualify for their service, despite the fact that the reason she left home that morning was because she had discovered that her mother was having sex with her boyfriend!

Perhaps this would not happen today? No, that can't be right as we have more 'non-service' delivery scandals than ever. Perhaps this wouldn't happen in the UK? Perhaps I'm just naive?

C. Cantor Psychiatrist, Noosa Heads, Queensland, Australia

\section{The interface between child and adult mental health services}

The divide between services for children and adults with mental health problems continues, so I was pleased to read the article by Singh et al (Psychiatric Bulletin, August 2005, 29, 292-294) which draws our attention to this matter again. However, I think that more emphasis should have been placed on the important role of training, particularly for junior psychiatrists and general practitioners (GPs) who will be in the vanguard of developing or commissioning services in the future.

With this in mind, I have started to run an induction session in child psychiatry for our child and adolescent mental health service (CAMHS) in Plymouth. This began as an hour but is now half a day and may shortly be a day-long event. It is intended for new senior house officers in psychiatry, who may be career psychiatrists or vocational GP trainees, and occurs every 6 months as part of their routine induction programme. The evaluation of these sessions has been very positive, with all trainees so far finding the sessions 'useful' or 'very useful'. This is the main reason that the length of the session will be extended: it seems to be filling a training need which is probably not met elsewhere. General practitioners not only have to deal with a considerable burden of psychiatric illness of both adults and children in primary care, but also receive very little training for this. Foreman (2001), for example, found that $47 \%$ of GPs sampled had no undergraduate training in CAMHS and $93 \%$ had negligible postgraduate experience.

The session includes an initial introduction to the CAMHS, followed by sections on self-harm and the local protocol for its assessment in young people, and the effects of parental mental illness on children. The second half of the session covers conditions commonly seen in a CAMHS which will continue into adult life, such as attention-deficit hyperactivity disorder and autistic-spectrum disorder. The teaching is interactive and videos provide a focus for discussion.

I would be interested to hear of other developments in CAMHS throughout the country on GP training in child and adolescent psychiatry. Perhaps the College should be developing an initiative to this end?

FOREMAN, D. M. (2001) General practitioners and child adolescent psychiatry: awareness and training of the new commissioners. Psychiatric Bulletin, 25 , 101-104.

S. Huline-Dickens Consultant in Child and Adolescent Psychiatry, Erme House, Mount Gould Hospital, Plymouth PL4 7QD, e-mail: Sarah. HulineDickens@phnt.swest.nhs.uk

\section{Ward rounds - patients' views}

We have similar concerns regarding patients' views of ward rounds to White \& Karim (Psychiatric Bulletin, June 2005, 29, 207-209). Our service is a low secure forensic unit, which provides long-term rehabilitation in the West Midlands, and in contrast to general adult services inpatients have a 4-weekly ward round slot.

A recent review of records of 12 inpatients over a 6-month period highlighted that patient attendance at ward rounds has been poor - 2 patients attended frequently, 6 occasionally and 4 never. White \& Karim fail to mention that standard nursing practice is to provide selective written and verbal feedback to patients after the ward round. Therefore the patients may feel that they do not need to attend as they receive comprehensive feedback without undergoing the ward round experience.

Hodgson et al (Psychiatric Bulletin, May 2005, 29, 171-173) stressed the compromise position of the ward round as it struggles to serve both professional and patient needs. The duties of a doctor according to the General Medical Council include the need to respect the rights of patients to be fully informed in decisions about their care, to give patients information in a way they can understand and to listen to patients. By maintaining the practice of ward rounds in which patients choose not to participate, are we failing to involve patients in decisions about their care? Patients want individual consultant time and ward rounds do not allow this. Perhaps the way forward is to have both a team meeting followed by individual patient time with a consultant.

*F. Hynes Specialist Registrar, West Midlands Forensic Psychiatry Rotation, Hillis Lodge, Hollymoor Way, Northfield, Birmingham B315HE, e-mail: fhynes@doctors.org.uk, O.G. Muchemenye Senior House Officer, Low Secure Forensic Rehabilitation Service, Birmingham

\section{Guidelines for prescribing injectable heroin and methadone}

I was surprised to read that heroin prescribing was considered controversial (Luty, 2005). In the 1970s my colleague and I had no serious problems prescribing heroin and cocaine. In the 1980s and '90s Dr John Marks successfully prescribed heroin in Widnes but there was great hostility to his programmes. I have not seen his success mentioned in official or clinical discussion, including the 2003 guidelines from the National Treatment Agency for Substance Misuse. He has been 'air-brushed' out of history. 